\title{
Statistical analysis of sunspot area and magnetic flux variations in 2001-2003
}

\author{
S. I. Zharkov and V.V.Zharkova \\ Department of Cybernetics, Virtual Systems and Internet, Bradford University, Bradford BD4 \\ 6ES, UK; email: s.zharkov@brad.ac.uk
}

\begin{abstract}
This paper utilizes a searchable Solar Feature Catalogue (SFC) for sunspots created from the SOHO/MDI whitelight images and magnetograms in 2001-2003 using the automated pattern recognition techniques described at http://www.cyber.brad.ac.uk/egso/. Comparison of sunspot areas taklen from the SFC with the daily sunspot numbers published in the Sunspot Index Data Centre (SIDC) shows very good correlation of $78-86 \%$. The total magnetic flux from sunspot areas, measured from a single solar image, is shown to have a strong northern-southern asymmetry revealing magnetic flux decrease in the northern hemisphere and an increase in the southern one with the solar cycle descendence towards the period end (year 2003). The resulting, or excess, flux has also demonstrated very significant asymmetry being negative in the southern hemisphere and positive in the northern at the beginning of the period (solar maximum, 2001)with its amplitude descreasing towards the the period end (2003), or the solar minimum. Also, in 2003 there are signs of change in the excess flux polarity between the northern and southern hemispheres.
\end{abstract}

\section{Introduction}

The averaged relative numbers are listed in the Sunspot Index Data Center (SIDC) in the World Data Center for Sunspot Indices (Rishbeth 1991; Ruttenberg \& Rishbeth 1994). With substantial increase in the size of solar image data sets, the automated detection and verification of various features of interest is becoming increasingly important for reliable forecast of solar activity and space weather and data mining.

Recently, the N-S asymmetry was detected in the derived monthly mean hemiospheric Sunspot Numbers, which was found to vary during solar cycle and from cycle to cycle (Temmer et al., 2002). The attempts to produce reliable statistical models of sunspot numbers have revealed substantial gaps in the solar activity knowledge which can be uncovered extracting and analyzing additional feature parameters. This became possible with the creation of the Solar Feature Catalogues (SFCs) (Zharkova et al., 2004) within the European Grid of Solar Observations project (Bentley et al., 2003).

\section{The data description and validation}

\subsection{The SOHO/MDI whitelight images and magnetograms}

We use the level 1.8 calibrated synoptic daily continuum and magnetogram MDI datasets available almost continuously from May 1996 until the present days with cadence of 4 continuum and 15 magnetogram observations per day. The continuum images were preprocessed/flattened by compensating limb-darkening following the cleaning technique described in Zharkova et al., 2003. The relevant magnetograms were smoothed with Gaussian (5x5) filter and synchronized to the time and point of view of the continuum observation. 

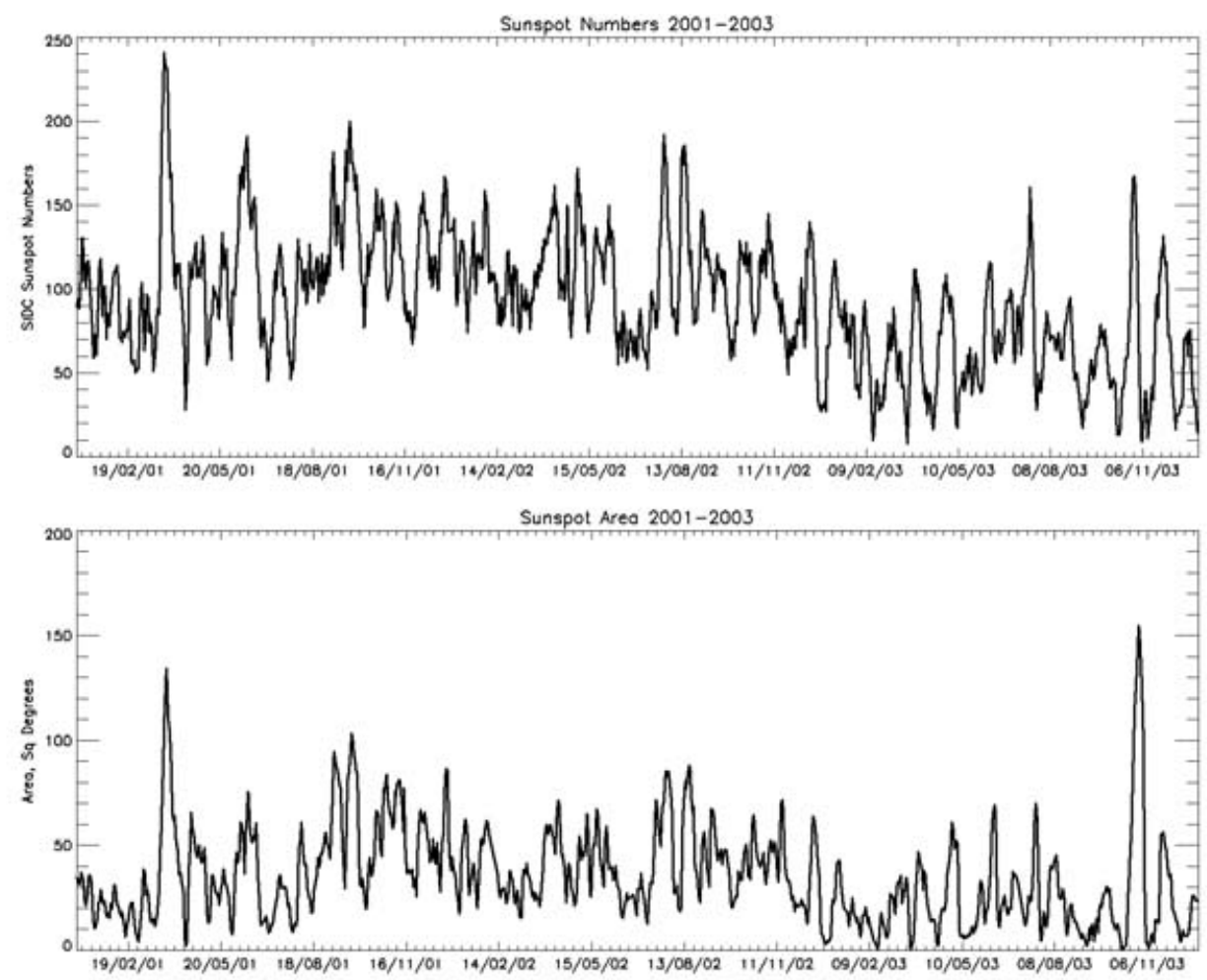

Figure 1. A comparison between SIDC sunspot numbers over the whole solar disk (the upper plot) with sunspot areas derived from the Solar Feature Catalogue of sunspots (the lower plot) for 2001-2003 with the correlation coefficient of 0.82 (see discussion in the text).

\subsection{The Solar Feature Catalogue for sunspots}

The sunspots were detected on full disk images using the automated technique based on edge detection and local thresholding as described by Zharkov et al. (2003, 2004). The sunspots are considered as features which are darker than the quiet Sun and characterized by strong magnetic field and well-defined borders. This approach allows us to detect smaller features like sunspot pores with reasonable degree of accuracy.

Once the sunspots are detected, the following information is extracted and stored in the SFC catalogue: sunspot gravity center Carrington coordinates, gravity center planar coordinates, heliographic area, heliographic diameter, number of umbras detected, maximum flux, minimum flux, excess flux, total (absolute) flux, maximum umbra flux, minimum umbra flux, excess umbra flux, total (absolute) umbra flux, the pixel and planar coordinates for the bounding rectangle, its raster scan marking the penumbral and umbral pixels as well as the observational parameters such as a date of observations, a solar radius, the disk center coordinates and image resolution (Aboudarham and Zharkov, 2004). These parameters are then inserted into mysql database which is published at Bradford University web-site and can be accessed at http://www.cyber.brad.ac.uk/egso/. 


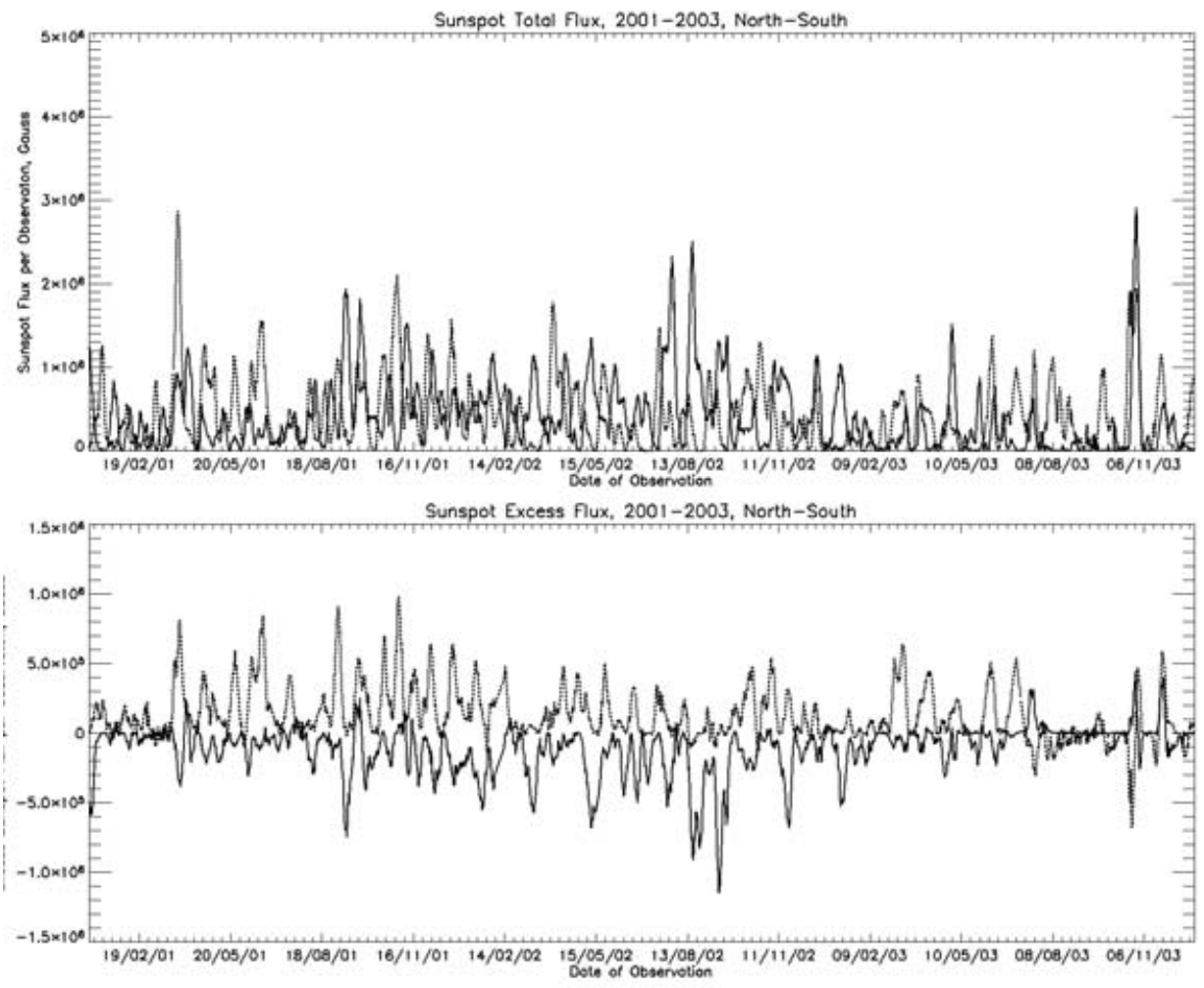

Figure 2. The North-South asymmetry: sunspot absolute magnetic flux (upper plot) and resulting, or excess, magnetic flux (the lower plot) for Northern (solid line) and Southern (dotted line) hemispheres.

\section{The results and discussion}

\subsection{The sunspot area variations}

As a means of verification let us compare the sunspot data from SFC with the averaged sunspot numbers presented in the Sunspot Index Data Center (SIDC) (Rishbeth 1991; Ruttenberg \& Rishbeth 1994). Sunspot numbers are not yet derived directly from the SFC data (Zharkov et al., 2004) the area of solar surface covered by the sunspots on a given day can be used as an indicator of the solar activity instead. For every image we calculate the sum of the areas covered by all sunspots detected in this image and define it as total area per observation. In Figure 1 the sunspot numbers taken from SIDC are presented versus time for the years 2001-2003 (the top plot) and the total sunspot areas of a solar surface (the bottom plot).

A comparison of the plots reveals that the temporal variations of both sunspot numbers and their areas appear to be very similar. In order to quantify this similarity, a crosscorrelational analysis was run for the both arrays, and the correlation coefficients were found to be equal to 0.86 for 2001, 0.82 for 2003 and 0.78 for 2003 producing 0.82 for the whole period 2001-2003. This indicates the very high correspondance between the SFC and SIDC data, while the correlation differences can be understood in terms of the different approaches used for these datasets. The SIDC data is biased towards the number of Sunspot Groups, which contributes with the factor of 10 for every observation, while the SFC set presents a total area covered by sunspots on a given day. Clearly, the two 
statistics will behave differently in a number of situations, such as appearance of a new sunspot group, which produces a quantative jump in SIDC number on that day and, in general, only a small increase in the SFC's areas. This explains the correlation coefficient between the statistics being smaller then 1 with both sets describing the same process. The automated classification of sunspots into groups, we believe, will further improve the correlation between these two sets and is a scope of the forthcoming paper.

\subsection{North-South asymmetry in magnetic fluxes}

By synchronising the continuum and magnetogram images, for every sunspots and for every image, one can estimate the magnetic flux over sunspot area. This allows us to extract the following parameters: total magnetic flux as a sum of the absolute magnetic field pixel values and excess, or reulting, magnetic flux measured as an algebraic sum of the magnetic field pixel values. Note, that in order to get the flux in $\mathrm{Mx}$, the magnetic induction values have to be multipled by the area of a single pixel that is 2"x2". Based on the sunspot location, these statistics can be sorted seperatelly for the northern and southern hemispheres. The results extracted for 2001-2003 are presented in Figure 2 for the total flux (upper panel) and excess flux (lower panel).

It can be seen that total magnetic fluxes in Northern and Southrn hemispheres have different patterns: the Southern one has larger areas in year 2001 (solar maximum) while the Northern hemisphere takes over in 2003 (towards the solar minimum). This pattern is even more pronounced in the excess flux where one can see a suprising full separation of the resulting flux in each hemipshere: in 2001 the Northern hemishpere excess flux has a pure negative polarity, with the Southern flux being positive nearly everwhere. However, towards the solar minimum (2003) one can see that this separation is violated so that the Northern hemisphere excess flux is changing to positive and southern to the negative. Since only 3 years were investigated, this result should be considered as a preliminary one that requires further investigation of sunspots and magnetic fields for the whole period of the available $\mathrm{SOHO} / \mathrm{MDI}$ data.

\section{Acknowledgements}

This research is supported by the European Grid of Solar Observations project funded by the European Commission within the IST Framework 5 the grant number IST-200132409 .

\section{References}

Aboudarham J. and Zharkov, S.S., http://www.cyber.brad.ac.uk/egso/SFC2/FeaturesParametershtml.htm, EGSO-WP5-IR3-2.1, 28p., 2004

Bentley , R.D. 2002, in Proc. 2nd Solar Cycle and Space Weather Euro-Conference, Vico Equense, September 2001 ESA SP-477, ESA Publications, 603-607.

Rishbeth, H. 1991, J. Geomagn. Geolelctr. Suppl., 43, 921

Ruttenberg, S. \& Rishbeth, H. 1994, J. Atm.Solar Terr. Phys. 56, 865.

Sherrer, P.H. et al., 1995, Ann. Rev. Astr.Astrophys., 2, 363

Temmer, M., Veronig, A. \& Hanslmeier, A. 2002 A \% A 390, 707-715.

Waldmeier, M. 1961, The sunspot activity in the years 1610-1960, Scultheiss Publisher, Zurich.

Zharkov, S.I.; Zharkova, V.V.; Ipson, S.S. and Benkhalil, A.K., 2004, Proc. KES2004, Wellington, New Zealand, p.584-590.

Zharkova, V.V., Ipson, S. S., Zharkov, S.I. and the other 3 authors 2003, Solar Phys. 214, 89-105.

Zharkova, V.V.; Aboudarham, J.; Zharkov, S.I.; Ipson, S.S.; Benkhalil, A.K. and Fuller, N., 2005, Adv.SpaceRes., subm. 